\title{
Does the extended evolutionary synthesis entail extended explanatory power?
}

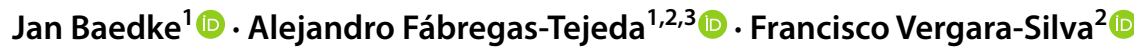

Received: 29 May 2019 / Accepted: 9 January 2020 / Published online: 23 January 2020

(c) The Author(s) 2020

\begin{abstract}
Biologists and philosophers of science have recently called for an extension of evolutionary theory. This so-called 'extended evolutionary synthesis' (EES) seeks to integrate developmental processes, extra-genetic forms of inheritance, and niche construction into evolutionary theory in a central way. While there is often agreement in evolutionary biology over the existence of these phenomena, their explanatory relevance is questioned. Advocates of EES posit that their perspective offers better explanations than those provided by 'standard evolutionary theory' (SET). Still, why this would be the case is unclear. Usually, such claims assume that EES's superior explanatory status arises from the pluralist structure of EES, its different problem agenda, and a growing body of evidence for the evolutionary relevance of developmental phenomena (including developmental bias, inclusive inheritance, and niche construction). However, what is usually neglected in this debate is a discussion of what the explanatory standards of EES actually are, and how they differ from prevailing standards in SET. In other words, what is considered to be a good explanation in EES versus SET? To answer this question, we present a theoretical framework that evaluates the explanatory power of different evolutionary explanations of the same phenomena. This account is able to identify criteria for why and when evolutionary explanations of EES are better than those of SET. Such evaluations will enable evolutionary biology to find potential grounds for theoretical integration.
\end{abstract}

Keywords Extended evolutionary synthesis · Standard evolutionary theory · Explanatory power $\cdot$ Explanatory standards $\cdot$ Developmental bias $\cdot$ Niche construction

Jan Baedke

jan.baedke@rub.de

1 Department of Philosophy I, Ruhr University Bochum, Universitätsstrasse 150, 44801 Bochum, Germany

2 Instituto de Biología (Jardín Botánico), Universidad Nacional Autónoma de México, Circuito Exterior Ciudad Universitaria S/N, 04510 Mexico City, Mexico

3 Posgrado en Filosofía de la Ciencia, Universidad Nacional Autónoma de México, Mexico City, Mexico 


\section{Introduction}

In the last decade a growing number of biologists and philosophers of science have argued that evolutionary theory needs to be 'extended' in order to explain evolutionary change better. They claim that the standard framework of evolutionary biology-especially the population genetics central to the modern synthesis-is insufficient to account for the diversity of evolutionary processes. According to this view, standard evolutionary theory (SET) should integrate more thoroughly new knowledge about how "developmental processes, operating through developmental bias, inclusive inheritance and niche construction share responsibility for the direction and rate of evolution, the origin of character variation and organism-environment complementarity" (Laland et al. 2015: 8; see also Pigliucci 2007; Mesoudi et al. 2013; Jablonka and Lamb 2014; Sultan 2017; Uller and Laland 2019). This novel framework is commonly called the 'extended evolutionary synthesis' (EES; see Pigliucci and Müller 2010a; Laland et al. 2014, 2015; Müller 2017). It draws especially on studies in evolutionary developmental biology (Evo-Devo sensu Müller 2007, which encompasses both evo-devo and devo-evo), epigenetics (Jablonka and Lamb 2014), and niche construction theory (sensu Odling-Smee et al. 2003). Proponents of EES are aware that the genuine integration of their evolutionary explanations under one novel, alternative theoretical framework is challenging, especially for linking developmental and evolutionary studies (Laland et al. 2013: 806).

In recent years, advocates of EES have tried to tackle this issue of integration. In particular, Kevin Laland, Tobias Uller, and colleagues have tried to clarify the explanatory content of EES by spelling out its distinct core assumptions and problem agenda. This content includes a novel explanatory focus on the unit of the organism, constructive development, and reciprocal causation of organism-environment interaction (Laland et al. 2015; for discussion, see Fábregas-Tejeda and Vergara-Silva 2018a; Baedke 2019). While these efforts have helped to clarify the explanatory aims and content of EES, we still lack precise knowledge about the explanatory standards of this conceptual framework and how they differ from those of SET. In other words, the EES debate has focused on question (1): What does EES seek to explain and which explanantia (e.g., developmental bias, epigenetic inheritance, niche construction) do the explaining? However, EES has neglected additional important questions: (2) What are its own explanatory standards? and (3) What actually makes explanations of EES better than those of SET? We claim that in order for the theoretical structure of EES to become more rigorously specified, questions (2) and (3) need to be addressed. Our paper meets this challenge by contrasting the standards of explanatory power among EES and SET, as well as evaluating the goodness of the explanations of the former compared to the latter. 
In general, supporters and critics of the importance of developmental processes for evolution agree that the third issue (i.e., which explanation is better and should be chosen) can and should be solved on empirical grounds (see Jablonka and Raz 2009; Wray et al. 2014). ${ }^{1}$ This means that a better explanation is simply the one that is better supported by available evidence. However, we are skeptical of the assumption that empirical data alone will settle the debate, and this skepticism is increasingly shared by evolutionary biologists in the field (see Uller et al. 2019). Recently, in evolutionary biology a number of 'standoff situations' between opposing EES and SET explanations have occurred, in which each explanation addresses the same (or very similar) phenomena with seemingly equal evidential support. Such standoffs have led to contrary interpretations of one and the same developmental phenomena (e.g., Laland et al. 2014; Wray et al. 2014; see Kovaka 2019). For instance, niche construction has been dismissed as nothing but an 'extended phenotype' (Scott-Phillips et al. 2014), or described as a causal starting point of evolutionary trajectories (Laland et al. 2005). Developmental plasticity has been explained as merely an adaptation to environmental variation or as facilitating and directing evolutionary processes (reviewed in Sul$\tan$ 2017; Uller et al. 2019). Finally, epigenetic inheritance has been accounted for as under genetic control (Dickins and Rahman 2012) or as an additional and (partly) independent source of variation (Jablonka 2017).

Given these standoffs between opposing explanations, it becomes important to understand not only how well each of these explanations is supported by evidence but also the nature of the explanatory standards that govern each of them. In other words, we argue that the question of EES's and SET's explanatory goodness is in need of a thoroughgoing investigation. We present a theoretical framework of explanatory power. It follows Woodward $(2003,2010)$ and Ylikoski and Kuorikoski (2010), and allows us to clarify why (i.e., according to which criteria) and when (in which explanatory contexts) one evolutionary explanation can be considered better than another.

We first introduce the explanatory aims and pluralist problem agenda of EES ("Pluralism and explanation in the EES"). Second, we present a general approach for evaluating the value of scientific explanations ("What explanatory power is and is not"). Third, by drawing on a case study on early plant domestication, we specify the criteria by which typical explanations of EES have more explanatory power than standard evolutionary explanations ("Evolutionary explanations of early plant domestication" and "Different explanatory standards in evolutionary biology"). We conclude that our conceptual framework allows the identification of differences in explanatory standards between EES and SET, and suggest such evaluation will enable future theoretical integration in evolutionary biology.

\footnotetext{
1 Or on methodological grounds (see Svensson 2018).
} 


\section{Pluralism and explanation in the EES}

Some evolutionary theorists have argued that EES should provide a comprehensive theory of phenotypic evolution that is different from the 'theory of genes' pursued by SET-oriented biologists (Pigliucci 2007). Pigliucci and Müller (2010b: 12) described this as a "shift of emphasis from statistical correlation to mechanistic causation." Such arguments have encountered both support and criticism (e.g., see Pennisi 2008; Dickins and Rahman 2012; Fábregas-Tejeda 2019). ${ }^{2}$ In the ensuing debate, some authors have presented EES as being in opposition to the populational framework of SET (Craig 2010; Noble 2011). ${ }^{3}$ This purported disagreement is epitomized by the famous debate in the journal Nature between teams with opposing views (Laland et al. 2014; Wray et al. 2014). For proponents of EES, the narrow and 'gene-centric' stance of SET fails to capture the "full gamut of processes that direct evolution" (Laland et al. 2014: 162). They hold that variation, heredity, and selection should not be always understood as causally autonomous, as the causes that sustain one component of evolution by natural selection may be intertwined with causes of other components through ontogenetic processes (see Baedke 2017; Uller and Helanterä 2019; Uller et al. 2019). Instead, processes such as developmental bias, phenotypic plasticity, extra-genetic inheritance, and niche construction should be understood as developmental 'proximate causes' that can direct and facilitate evolutionary change. A central epistemic goal for EES is thus to provide mechanistic explanations of the origin and maintenance of functional and selectable phenotypic variation. ${ }^{4}$ Another epistemic goal is to understand the constructive roles organisms play in shaping their own development and evolution, and how they change their environments in evolutionary meaningful ways (e.g., through niche construction) (Fábregas-Tejeda and Vergara-Silva 2018a).

For advocates of EES, there is an overall agreement that this conceptual framework offers a more pluralist perspective to understand and explain evolutionary phenomena when compared to SET (see quotes 1-4 in Table 1). This greater pluralism (see Tanghe et al. 2018) has two dimensions: the introduction of (a) new explananda and (b) better explanations for explananda already addressed by SET. With regards to (a), EES is said to explain phenomena neglected by SET (see quotes 2, 5-6 in Table 1). With respect to (b), the pluralism of EES is said to explain more completely the same evolutionary phenomena by addressing them from different explanatory angles or with different epistemic goals (e.g., by embracing a developmental standpoint; see quotes $6-8$ in Table 1 ).

The pluralist contributions of EES ( $a, b)$ are usually considered to increase the explanatory power of evolutionary theory (e.g., Peterson and Müller 2016: 328). In contrast, critics of EES doubt this is achieved or even needed. For example, Wray et al. (2014: 163) argue that "none of the phenomena championed by Laland and

\footnotetext{
${ }^{2}$ We should stress here that within both 'camps' there is a wide diversity of views. Thus, labeling views as SET or EES, or branding individuals as advocates or critics of EES, to some extent, risks distorting complex scientific practices and perspectives (see also Lewens 2019).

3 This can be misleading, however, as many EES-related research (e.g., in population epigenetics) adopts this SET-framework.

${ }^{4}$ On the notion of epistemic goals, see Brigandt (2010) and Love (2010).
} 


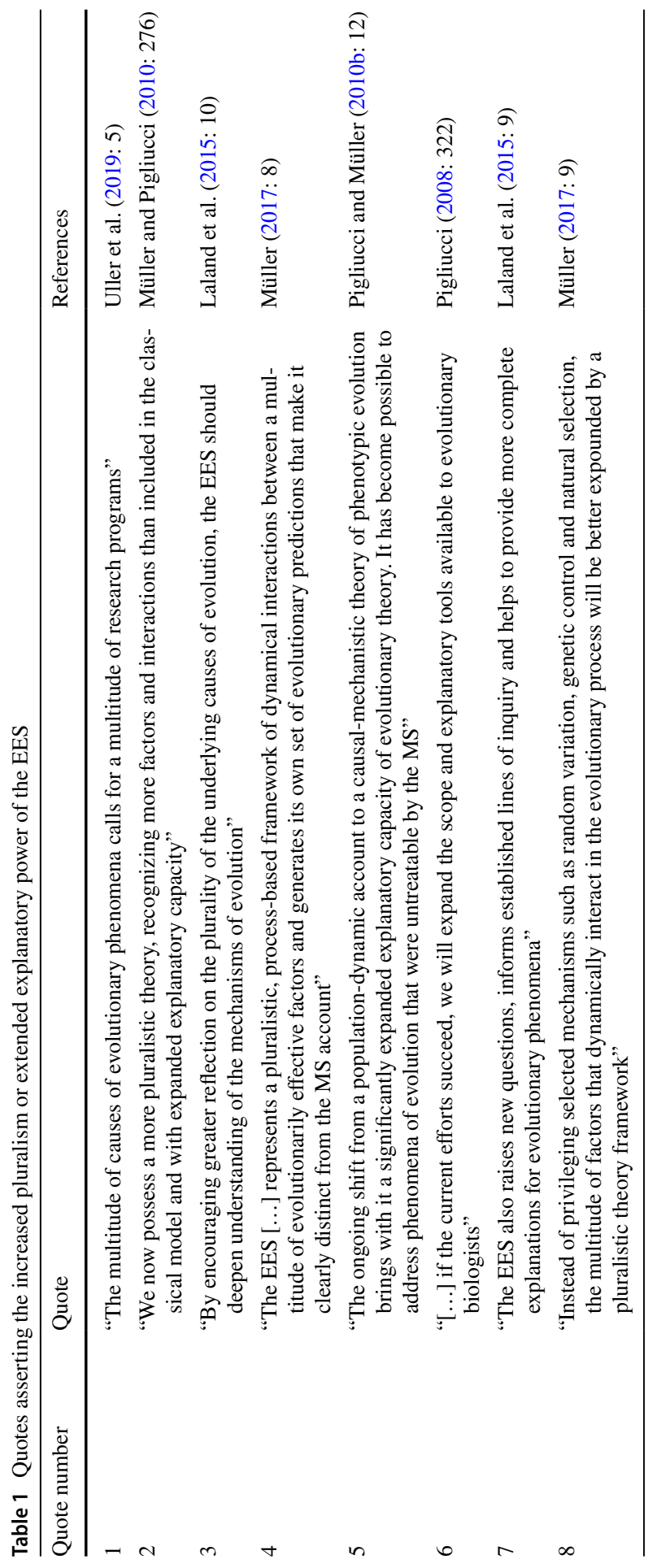


colleagues are neglected." Instead, their inclusion in SET "reflects their proven explanatory power, not a lack of attention." Given these contradictory views, we need to understand how advocates of EES and SET evaluate the explanatory power of their approaches. Do they do so based on evidential support alone, or do they apply different standards of explanatory goodness? More specifically, what are the criteria that EES and SET apply when saying their explanations are good or better than the other side? In order to evaluate the relative merits of EES's and SET's explanations beyond their empirical basis, we present a conceptual framework of explanatory power.

\section{What explanatory power is and is not}

While evolutionary explanations drawing on developmental processes such as developmental bias, epigenetic inheritance, and niche construction have become increasingly common, it is far from clear why these new explanations are better and thus should be chosen over more orthodox explanations. This problem is related to the concept of explanatory value or power. ${ }^{5}$

In the EES debate, the idea of explanatory power plays a central role. EES supporters not only want their claims to be accepted by defenders of SET as valid scientific explanations, but they want them to be recognized as complementary (or even superior) alternatives that lead to a "significantly expanded explanatory capacity" for evolutionary theory (Pigliucci and Müller 2010b: 12). Such claims about EES's greater explanatory power are usually accompanied by calls to better integrate novel developmentalist explanations with SET-based evolutionary explanations. In this pluralist theoretical context, we need to develop reliable criteria for why and when we should choose one explanation over others. In other words, explanatory power itself needs specifying. We go beyond the usual candidates of likeliness and causal power.

Likeliness: Assessing the explanatory power of EES should not be equated with evaluating its evidential power or likeliness (i.e., when one explanation is supported by more evidence than another). Likeliness has been a cornerstone of the EES vs SET debate, both for revisionary (Jablonka and Raz 2009; Laland et al. 2015; Müller 2017) and more orthodox approaches (Wray et al. 2014; Futuyma 2017). Nevertheless, the issue of empirical support differs from the question of which explanations should be favored once there is evidential parity between EES and SET (or when both are similarly well supported). ${ }^{6}$ Thus, likeliness is an insufficient criterion. When similar levels of evidential support still result in standoffs between two

\footnotetext{
${ }^{5}$ Explanatory power should not be mixed up with the so-called demarcation problem, i.e., distinguishing scientific from non- or pseudoscientific explanations, nor with criteria for identifying scientific explanations, traditionally discussed in philosophy of science, like predictive capability, evidential support, parsimony or simplicity, a particular formal structure, and coherency or compatibility with background knowledge. We will defend a concept of explanatory power that moves beyond the above criteria while still regarding them as genuine criteria of scientific explanations.

${ }^{6}$ While not discussed in detail here, evidential parity of two competing explanations that address the same phenomenon can be assessed quantitatively. For instance, Bayesian approaches to evidential support allow formalizing the degree of confirmation of competing hypotheses (Crupi et al. 2007).
} 
alternative explanations, their value must be distinguished on other grounds. Advocates of EES refer to this problem when they ask "what makes an evolutionary explanation more or less satisfactory?" rather than settling just for how likely it is (Uller et al. 2019: 7).

Causal power or importance: Explanatory power is often conflated with the question of whether an explanation includes more important causes than another. This ontological idea rests on the assumption that all the causes relevant for a phenomenon under study share objectively measurable or comparable properties. This view guides so-called 'causal democracy' claims in developmental systems theory (Oyama et al. 2001). Such claims have been influential for EES's causal reciprocity thesis and niche construction theory (Laland et al. 2011, 2016). Causal democracy theory argues that rather than privileging certain causes (e.g., genes), every factor involved should initially be approached as causally important. Subsequently, explanations should cite only causes with objectively measurable high causal power. Although providing a balance to gene-centrism, developmental systems theory takes for granted that listing the most important causes automatically increases an explanation's value. However, this is not necessarily the case. Two different explanations of the same phenomenon based on different sets of causes with equal causal importance might not be equally good (see Sober 1988).

The notion of explanatory power we defend goes beyond these two concepts of likeliness and causal power. ${ }^{7}$ We hold that evaluations of explanatory power should be made when there are different (and possibly opposing) explanations that are accepted by relevant epistemic communities as valid scientific explanations (e.g., based on their likeliness), but that differ in their underlying explanatory standards. Our specification of explanatory power draws on the interventionist framework of explanation (Woodward 2003). In this framework, explanatory regularities should remain invariant under an intervention (i.e., a manipulation of a value in an explanans variable would lead to a change in the value of an explanandum variable). Such invariant regularities answer counterfactual w-questions (i.e., what-if-things-hadbeen-different-questions) on what would happen to an explanandum variable if one would intervene on one or more explanans variables. An interventionist approach shows how achieving particular criteria of explanatory power affects the degree of invariance of an explanation and thus its information value (Woodward 2010; Ylikoski and Kuorikoski 2010). This means that explanatory power can be assessed by comparing the range of inferences to potentially new counterfactual situations and, accordingly, of answers to w-questions that alternative explanations make possible. A better explanation provides more information on patterns of counterfactual dependency. For example, an explanation of a particular population dynamic that

\footnotetext{
7 At the same time, we distance ourselves from the assumption that evolutionary explanations are selected irrationally and (largely) independently of their evidential support (Haig 2011; Welch 2017). We will show that the value of evolutionary explanations can be compared rationally.
} 
includes developmental factors might be able to answer more questions on what would happen to this population if it was changed. If it can answer more of these questions than an alternative explanation that does not include these developmental factors, the former carries more explanatorily relevant information. By interventionist lights and our own view, this explanation is therefore better.

Such comparisons are easier if we are very precise about the relevant explanatory relations. We will use an account of explanation in which both explanans and explanandum are contrastive (Woodward 2003; Schaffer 2005; Ylikoski and Kuorikoski 2010; Raerinne and Baedke 2015). The contrastive account represents the claim " $x$ rather than $x *$ explains $y$ rather than $y^{*}$ in context $U$ " as " $x\left[x^{*}\right]$ explains y $\left[y^{*}\right]$ in context $U$," whereas $x$ and $y$ represent the explanans and explanandum, respectively, and $x^{*}$ and $y^{*}$ their contrast classes. The context $U$ describes the system's other properties and environment. Now we can evaluate how achieving particular explanatory virtues affects the invariance of a contrastive explanation and, consequently, its power. To do this, we focus on the explanatory standards of precision, proportionality, sensitivity, and idealization (Woodward 2010, 2018; Ylikoski and Kuorikoski 2010). ${ }^{8}$ There are important trade-offs between them that affect an explanation's value.

The degree of precision of an explanation refers to the amount and specificity of detail that is included into the explanans, explanandum, and contrast classes. Such detail can be gathered from various levels of biological organization. Often it is gathered from lower levels (e.g., molecular-genetic) in order to address a higher-level phenomenon. Precision has been linked, for instance, to the question of whether natural selection can merely explain the general dynamics of trait frequencies and survival or also the development of particular traits of individuals (Sober 1984; Neander 1995). According to the interventionist conceptual framework we are using, a more precise explanation entails more explanatorily relevant information, because it allows more and possibly important inferences to counterfactual scenarios, and thus answers new w-questions. ${ }^{9}$ A more precise explanation thus has more explanatory power and leads to better understanding. This is why some developmentally-minded evolutionary biologists suggest that evolutionary change should be explained not only by natural selection, but also by taking more developmental, physiological, or behavioral features and contrast classes into account (Uller et al. 2019: 5-6). Nonetheless, more precision does not amount on its own to better explanations. In fact, precision can also be misleading, such as when it adds irrelevant information to an

\footnotetext{
8 Ylikoski and Kuorikoski (2010) discuss further criteria, such as the degree of integration into a larger body of knowledge or cognitive salience. While we exclude these criteria for reasons of simplicity, in principle, our discussion of explanatory power can be expanded by considering these criteria as well. On the challenges that different evolutionary explanations face when integrated into one theoretical framework, see the concluding section.

9 Precision is connected to the idea of specificity of an explanation. A causal explanation can be described as specific if it not only explains that $X$ causes $Y$ but also provides precise details about which exact changes to $X$ would be associated with changes in $Y$ (Woodward 2010; see also Griffiths and Stotz 2013: ch. 4; Baedke 2018: ch. 5).
} 
explanation (see Nathan 2012). Besides this, precision shows important trade-offs with other explanatory standards, as we will show below.

Another criterion for saying that explanations are better argues that they should cite causes proportional to their effects, meaning that they have the same degree of precision and thus level of analysis (Yablo 1992; Woodward 2010, 2018). ${ }^{10}$ For instance, if an explanation cites a cause that is too detailed or specific, it may not be proportional to its effect (see Blanchard 2018). More generally, a proportional explanation conveys accurate information on the right level of analysis about alternative states of the effects by citing only causes that are sufficient and required for the effect. In the EES vs SET debate, assumptions about proportionality and the right level of explanation are central. EES advocates argue that SET misses important developmental causes when explaining evolutionary processes (Laland et al. 2015). In contrast, more orthodox evolutionary biologists argue that only "natural selection, drift, mutation, recombination and gene flow" are the necessary and required (and thus proportional) causes for explaining "the heritable differences in traits, especially those that bestow some selective advantage" (Wray et al. 2014: 164).

Besides these effects of precision on explanatory proportionality, a highly detailed explanatory relationship has more sensitivity (or is less stable) to changes in values of explanans and explanandum variables or of background conditions. In other words, it continues to hold only under a small number of these changes. In contrast, a less sensitive explanatory relationship is more powerful, as it allows inferences to more counterfactual situations in which variables (or background conditions) take on new values without breaking the relationship. Such less sensitive regularities are better as they give answers to more w-questions. ${ }^{11}$

Importantly, precision is often decreased through idealization. Idealized explanations distort the values of certain relevant factors to some false but easily managed extremes (e.g., 0, 1, infinity). Such distortions allow complex phenomena to be grasped (Potochnik 2017). For instance, advocates of EES have argued that representing plasticity as a reaction norm (i.e., the response of an expected phenotype of a given genotype to environmental conditions) conforms with the idealizations of selective explanations (i.e., those based on fitness differences). This representation, however, "can give the false impression that plasticity has no explanatory power for adaptive evolution" (Uller et al. 2019: 1). The tradeoff is that idealized explanations are usually not very precise, so they allow for relatively stable inferences to counterfactual situations. Highly idealized explanations (e.g., selective fitness based accounts on the costs and benefits of alternative phenotypes) provide incorrect answers to detailed w-questions and thus generate incomplete understanding. In contrast, explanations of adaptive evolution that include developmental phenomena, like phenotypic plasticity, allow for more w-questions to be answered. For example,

\footnotetext{
${ }^{10}$ For criticism of Yablo's and Woodward's view of proportionality, see Franklin-Hall (2016). For a defense, see Woodward (2018).

11 Note that this does not mean that a broken dependency relation provides no explanatory relevant information about the phenomenon at all. For instance, against Woodward (2003), it has been argued that explanations of robustness in biology rely on such cases of failed invariance under intervention (Baedke 2018: ch. 4).
} 
they show in detail how developmental mechanisms account for the phenomenon that some traits exhibit adaptive variation more likely than others (see Uller et al. 2019).

Similarly, Love (2018) has argued that in developmental biology, the pursuit of generality and completeness lead to an explanatory trade-off. General explanations (citing only highly conserved molecular-genetic mechanisms) are more valued by developmental biologists than integrated and more complete explanations combining genetic and cellular-physical mechanisms, which tend to be more variable. When translated into our contrastive framework, generality can be linked to the standards of sensitivity (or stability) and idealization, whereas completeness translates to precision. In the cases Love (2018) mentions, low sensitivity offers developmental biologists the possibility of making highly generalizable inferences to more counterfactual situations without breaking the explanatory relationship.

Now that we have marshalled the various aspects of explanatory power (precision, proportionality, sensitivity, idealization), we will use them as a conceptual apparatus to evaluate the power of different explanations within the EES versus SET debate. We will discuss contrasting explanations of the evolution of early plant domestication and evaluate which of them have more explanatory power.

\section{Evolutionary explanations of early plant domestication}

The literature on the domestication of plants provides good examples of EES explanations clashing directly with SET explanations. Following Zeder (2015: 3191), we characterize domestication as a multigenerational, mutualistic relationship in which one species (Homo sapiens) influences the reproduction and care of another species. This relationship gives the partner organism an advantage over individuals that remain outside the symbiosis. The set of traits pertaining to the 'domestication syndrome' of crops includes seed retention, increased fruit and seed size, changes in branching habit and stature, modifications in reproductive strategies, and variations in secondary metabolites (Meyer et al. 2012: 31). To narrow down this case study, we focus on SET and EES explanations of the initial stages of plant domestication.

According to SET, the early domestication of plants hinges on human behavioral ecology in the form of 'optimal foraging theory'. Human behavioral ecology studies how measurable ecological variation predicts variation in behavioral fitnessenhancing strategies (Nettle et al. 2013). The "adaptationist stance" of optimal foraging theory holds that selection favors genes that contribute to optimal behaviors of individuals (Nettle et al. 2013: 1032). A widely held version of that theory is the 'diet breadth model', which states that foragers make decisions based on the energy returns of gathering resources compared to energy expenditures (Hawkes and O'Connell 1992). Optimal foraging thus emphasizes that human individuals should only exert selective pressures on the progenitors of domesticates during times of population-resource imbalance. These pressures allow humans to acquire more profitable environmental resources with selective advantages (Weitzel and Codding 2016). This model can be backed up by standard population genetics (see GuerraGarcía and Piñero 2017). 
Ultimate cause explanations in SET seek to answer the question of what a phenotype evolved for (Mayr 1961). In the plant domestication case as explained by 'diet breadth model', domestication arises as an adaptive response to an impoverished environment. In contrast, EES explanations cite additional developmental causes in order to answer how a character evolved (Laland et al. 2011). In the plant case, environmental changes may have directly caused phenotypic change in wild ancestors of crops by means of phenotypic plasticity (Mueller et al. 2017: 3-4). In this developmental process, epigenetic factors such as methylation differences play an important role (Piperno 2017). From an EES standpoint, domestication is thus seen as a "multi-generational coevolutionary relationship between two niche-constructing species with each undergoing changes (either genetically driven or plastic) that enhance the benefits each derives from the relationship" (Zeder 2017: 4). These interactions and phenotypic changes are actively mediated by organisms that engineer their own developmental niches with intergenerational consequences (Sterelny 2011).

Contrary to SET and its optimal foraging and diet breadth model, EES holds that cultural niche construction should be seen as "an important driver of evolutionary change that does not require resource depression to be set into motion" (Zeder 2015: 3196). By assuming some influence over the care and reproduction of a particular domesticate, the domesticator engages in niche-constructing activities aimed at increasing the supply or predictability of a resource. The domesticate, reciprocally, partakes in niche construction activities that secure fitness-enhancing benefits from its association with humans. We can outline these contrasting explanations of SET and EES as $\mathrm{E}_{\mathrm{SET}}$ and $\mathrm{E}_{\mathrm{EES}}:^{12}$

$\mathrm{E}_{\mathrm{SET}}$ : Optimizing energetic returns in times of resource imbalance was a primary causal factor in early domestication of plants. Selection favored genes in humans who were prone to behave optimally by exerting selective pressures on the progenitors of domesticates. Genes of plants causally associated with desirable traits (e.g., 'domestication syndrome' traits) were selected or changed in frequency through classic evolutionary forces in populations.

This evolutionary explanation involves the identification of loci that are statistically linked to 'domestication syndrome' traits. These range from specific transcription factors to metabolic enzymes (Meyer and Purugganan 2013). $\mathrm{E}_{\mathrm{SET}}$ traces how these alleles changed in frequency over generations through selective pressures, stochastic fluctuations, and other standard genetic changes. Now consider the alternative explanation:

$\mathrm{E}_{\mathrm{EES}}$ : Early domestication began as a co-evolutionary relationship between two active niche-constructing species that biased the selective pressures acting on them. As a consequence, both underwent heritable changes through

\footnotetext{
${ }^{12}$ We must recognize that there have been efforts to reconcile some elements of both explanations (e.g., Mohlenhoff et al. 2015).
} 
phenotypic plasticity and other ontogenetic processes. These changes led to 'domestication syndrome' traits that yielded mutual benefits to both species.

According to $\mathrm{E}_{\mathrm{EES}}$, plastic phenotypes responsible for the development of 'domestication syndrome' traits can be inherited. Genetic accommodation can occur if environmentally induced phenotypes are subsequently stabilized and fine-tuned across generations by selection (Laland et al. 2015). This is a plausible scenario for plant domestication, as has been recently argued by evolutionary archaeologists (Piperno 2017; Zeder 2017, 2018).

Although both $\mathrm{E}_{\mathrm{SET}}$ and $\mathrm{E}_{\mathrm{EES}}$ draw on supporting evidence of various kinds (e.g., archaeobotanical, genomic), defenders of cultural niche construction have argued that optimal foraging (and thus $\mathrm{E}_{\mathrm{SET}}$ ) lacks empirical support in many important centers of domestication (Smith 2015). The constructionist explanations of $\mathrm{E}_{\mathrm{EES}}$ have in turn been portrayed as untestable particularistic just-so stories that lack proper empirical support (Gremillion et al. 2014). As often seen in the SET versus EES debate, the result is a series of claims and counterclaims in plant domestication research that seemingly cannot be settled on empirical grounds alone. Such situations motivate us to turn to explanatory standards.

\section{Different explanatory standards in evolutionary biology}

In this section, we identify the criteria of explanatory power that are employed by developmentalist and adaptationist explanations of early plant domestication. We begin by analyzing the precision of each explanation. $\mathrm{E}_{\mathrm{SET}}$ focuses on genes and populations and abstracts away from details on the organismic level. $\mathrm{E}_{\mathrm{EES}}$, however, is supported by data collected from the molecular to the organismal level, and includes mechanisms of evolvability, phenotypic plasticity, pleiotropy and hybridization (reviewed in Zeder 2017, 2018). As a consequence, $\mathrm{E}_{\mathrm{SET}}$ and $\mathrm{E}_{\mathrm{EES}}$ differ with respect to the precision of their explananda, and their contrast classes:

Explanandum of $\left(\mathrm{E}_{\mathrm{SET}}\right)$ \{early domestication of plants\} [no domestication of plants]

Explanandum of $\left(\mathrm{E}_{\mathrm{EES}}\right)$ \{timing $\mathrm{t}_{1}$ and/or sequence $\mathrm{S}_{1}$ of early domestication of plants\} [different timing $t_{1+n}$ and/or sequence $S_{1+n}$ of early domestication of plants]

$\mathrm{E}_{\mathrm{SET}}$ enables evolutionary biologists to account only for a limited range of the contrast space relative to the explanandum of early domestication. It can answer why the relevant characters evolved (i.e., what were their selective payoffs), rather than why they did not. In contrast, $\mathrm{E}_{\mathrm{EES}}$ approaches generate a greater variety of answers to w-questions. For instance, "What would happen to 'trait x' of a domesticate if one or many causal factors involved in the developmental process changed to non-actual values?" Answering such questions is a fruitful scientific enterprise for understanding the occurrence and transgenerational persistence of traits along a number of quantitative parameters, such as the exact timing or sequence of plastic changes in plant development that might lead to domestication. 
For instance, in order to estimate quantitatively the 'environmental sensitivity' of natural living populations of teosinte, Piperno et al. (2015: 67) grew seeds in different conditions. They reproduced specific atmospheric $\mathrm{CO}_{2}$ values and temperature levels of the late Pleistocene, early Holocene, and modern-day conditions. Only in the first two (i.e., the assumed early domestication environments), but not in the third, was an invariant relation found between specific environmental conditions and the development of maize-like vegetative architecture and domestication traits. In short, they identified the range and threshold of environmental interventions under which early domestication could have occurred with a specific tempo: "We demonstrated here major phenotypic changes in one generation produced solely through the manipulation of environmental conditions" (Piperno et al. 2015: 72; emphasis added; see also Piperno 2017).

In the case of early plant domestication, we see that EES explanations do better than SET ones according to the explanatory standard of precision. EES can provide information about cultural niche construction, social learning, sources of variability, directionality, causality, modes of inheritance, targets of selection, and tempo of evolution (Zeder 2018). When assessed within our conceptual framework of explanatory standards, SET explanations should not be favored when, due to explanatory or methodological reasons, precision is the key criterion of explanatory goodness. The $\mathrm{E}_{\mathrm{SET}}$ adaptationist 'what for' explanandum shows less sharpness and thus requires an explanans with less precision. Its explanatory information provides only a limited range of inferences to counterfactual situations in which variables take non-actual values. Purely adaptationist explanations $\left(\mathrm{E}_{\mathrm{SET}}\right)$ thus provide a more limited understanding of evolutionary processes than broader developmentally informed explanations $\left(\mathrm{E}_{\mathrm{EES}}\right)$.

How do they fare when examined in light of the explanatory standard of idealization? The optimal foraging and diet breadth explanations of $\mathrm{E}_{\mathrm{SET}}$ are usually highly idealized because they deliberately distort explanans variables of human rationality, agency and behavior, as well as genotype-phenotype maps (in both plants and humans). These models assume that the behavior of organisms is close to optimal in terms of maximizing fitness, and that selection favors genes that contribute to individuals behaving optimally. And even though SET-minded scholars grant the existence of plasticity, many believe that "the capacity for plasticity is ultimately dependent on genotype, and plasticity is deployed in the service of genetic fitness maximization" (Nettle et al. 2013: 1032). In addition, the diet breadth model includes further idealizations (see Bettinger 1993: 84; Lins Neto and Albuquerque 2018). These are all elaborations of more general details in optimal foraging: The amount of time and energy needed to gain an energetic benefit from a given nutritional are usually fixed, and preferences for discrete food types are calculated in terms of higher momentary return rates only. Moreover, the selective environment is idealized in optimal foraging as a one-way, external force driving human domesticating behaviors (Smith 2015).

In contrast, $\mathrm{E}_{\mathrm{EES}}$ examples are less idealized because they usually distort the values of explanatory relevant causal factors to a smaller extent. In cultural niche construction, humans are not assumed to be perfectly rational, fitness-maximizing agents that always behave optimally in relation to fixed return rates. Instead, early 
domestication is thought of as a co-evolutionary relationship between two active species: humans are not optimal agents that dictate the evolutionary trajectories of plants because human behavior is also affected by reciprocal interactions with them. Likewise, organisms and environments are understood as entangled in reciprocal causal loops that shape evolutionary outcomes. Similarly, the genotype-phenotype relationship is less idealized. Humans and plants are treated as reactive developmental systems undergoing plastic changes on multiple levels of organization through non-linear pathways and various causes (Piperno 2017; Zeder 2017, 2018).

In sum, $\mathrm{E}_{\mathrm{EES}}$ scores better than $\mathrm{E}_{\mathrm{SET}}$ if we focus on the criterion of idealization and believe that less idealized explanations are better. However, it is too soon to conclude that less precise and more idealized $\mathrm{E}_{\mathrm{SET}}$ adaptationist explanations are always worse when compared to $\mathrm{E}_{\mathrm{EES}}$ developmentalist explanations. There is in principle no reason why the contrast classes of explananda addressed by adaptationist explanations could not be made more precise in future endeavors. Second, there are important trade-offs between these criteria. When precision is emphasized, sensitivity usually increases. Less precise, highly idealized explanations often allow more stable explanations (i.e., counterfactual relations are less easily disrupted though interventions).

For instance, according to Schlichting and Wund (2014: 666), in studies of phenotypic plasticity and epigenetic marking for a single system, the gold standard is to understand the complete set of links between environmental variation, developmental responses, and evolutionary changes to genetic and epigenetic architecture. An EES explanation of the evolution of 'domestication syndrome' traits would need to include all those factors, plus a characterization of niche constructing activities and changes in selection pressures and developmental niches, as well as any extra-genetic channels of inheritance in the reconstitution of phenotypes, and developmental processes that bias both the production of variation and trait selection. These detailed dependency relations are easily disrupted. They are more sensitive to changes in background conditions and changes to values of explanans variables (i.e., in developmental processes). Conversely, a less precise and more idealized SET explanation is more stable in that it enables inferences to more counterfactual situations without breaking the dependency relation. According to our framework then, the more robust $\mathrm{E}_{\mathrm{SET}}$ should be favored over $\mathrm{E}_{\mathrm{EES}}$ when explaining early plant domestication, when the explanatory standard is low sensitivity.

The final criterion to assess against our plant domestication example is proportionality. An EES developmentally-informed explanation of early plant domestication is proportional if it conveys accurate information, and only that information, about the conditions under which alternative states of the effect will be realized (Woodward 2010, 2018). To decide whether this is the case, let us restate the explananda of $\mathrm{E}_{\mathrm{SET}}$ and $\mathrm{E}_{\mathrm{EES}}$ :

Explanandum of $\left(\mathrm{E}_{\mathrm{SET}}\right)$ \{early domestication of plants [no domestication of plants]

Explanandum of ( $\left.\mathrm{E}_{\mathrm{EES}}\right)$ \{timing $\mathrm{t}_{1}$ and/or sequence $\mathrm{S}_{1}$ of early domestication of plants [ [different timing $\mathrm{t}_{1+\mathrm{n}}$ and/or sequence $\mathrm{S}_{1+\mathrm{n}}$ of early domestication of plants]. 
$\mathrm{E}_{\mathrm{EES}}$ achieves proportionality because it cites a precise set of specific environmental and developmental variables that are able to cause with a high probability the specific timing and sequence of appearance of early domestication traits (as shown by Piperno et al.'s experiments). It provides an analysis that does not suggests the importance of other implicit causes, such as genetic changes. According to our framework, high degrees of precision and limited idealization in both the explanans and explanandum guarantees the proportionality of $\mathrm{E}_{\mathrm{EES}}$.

However, $\mathrm{E}_{\mathrm{SET}}$ also connects causes and effects in a proportional manner. It simply uses a different strategy to achieve this explanatory virtue. $\mathrm{E}_{\mathrm{SET}}$ provides necessary and sufficient causes (especially natural selection) on the right levels of analysis to address a rather general effect, i.e., whether early domestication does or does not occur. Low degrees of precision and high levels of idealization in both the explanans and explanandum guarantee the proportionality of $\mathrm{E}_{\mathrm{SET}}$. Regarding the criterion of proportionality, both EES and SET explanations are equally good, although they follow different strategies to fulfill this standard. Nonetheless, this equal achievement does not hold when the less precise optimal foraging model seeks to explain the specific timing or sequence of the early domestication of plants. In other words, adaptationist explanations addressing the EES explanandum are less proportional. ${ }^{13}$

We consider this case of early plant domestication as representative of many of the oppositional situations in which EES and SET find themselves explanatorily. EES fares better with respect to precision, and SET shows less sensitivity and higher idealization. Both accounts have high proportionality, but each achieves this via different explanatory strategies. From the perspective of sensitivity, EES developmentalist explanations should not be preferred over traditional, less precise 'benefit' explanations, such as those derived from a diet breadth model. ${ }^{14}$ Therefore, in many cases where the explanatory context values low sensitivity over high precision, adaptationist explanations that are abstract and highly idealized but less sensitive may rightfully claim more explanatory power than competing EES explanations.

More generally, our analysis stresses the case-by-case evaluation of explanatory power according to the criteria of precision, sensitivity, idealization and proportionality. Neither SET nor EES explanations can be said to have more explanatory power in any a priori sense. Nevertheless, it must be recognized that EES developmentalist explanations have some explanatory power with respect to biological evolution. In short, EES explanations are genuine evolutionary explanations. Indeed, if the necessary explanatory virtues are high precision and low idealization, EES is the better theoretical approach to adopt.

\footnotetext{
13 Conversely, the same holds for developmentalist explanations addressing the SET explanandum.

14 However, counterexamples to this assessment outside of plant domestication research may exist (e.g., a developmentalist explanation that is less precise than a competing adaptationist explanation).
} 


\section{Advantages of this framework for the EES}

Many evolutionary biologists and theorists believe that we need to know the limits of any particular explanatory strategy, including the one championed by EES (e.g., Moczek 2019: 24). We completely agree. More specifically, we argue that in order to incorporate new and old explanatory approaches within a joint framework, analyses of explanatory power, such as the one presented here, are invaluable. Our framework allows researchers to assess the goodness of different evolutionary explanations in light of clear criteria. We have emphasized universal values of precision, proportionality, sensitivity, and idealization, as well as the trade-offs between them. Against this background, one can specify how opposing explanations could potentially be combined in order to lead to a better overall understanding of the phenomena to be explained.

As well as providing tools for comparing and assessing contrasting explanations, our framework helps to understand why in evolutionary biology there is often agreement over the existence of certain phenomena but disagreement about their explanatory relevance. Our motivation for applying this framework derives directly from the growing number of cases in evolutionary biology in which prevailing, adaptationist explanations of a particular phenomenon face opposing and seemingly incompatible explanations that draw on developmental mechanisms. Besides the evolution of early plant domestication discussed here, some other explanatory standoffs in evolutionary biology addressing the same or a similar explanandum are listed in Table 2. All of these cases and many more could be investigated with our theoretical framework of explanatory power.

Our view is that in many of these standoffs, each of the opposed explanations is partially correct, because natural selection and developmental processes together shape evolutionary outcomes (see also Laland et al. 2015; Uller et al. 2018, 2019). For example, in many instances, phenotypic plasticity is both an adaptive effect to fluctuations in environmental conditions and a developmental cause that affects the availability of variation and thus population dynamics. While we believe that all of the cases listed in Table 2 would profit from more evidential support, we are skeptical that there are empirical grounds that will adjudicate whether one of the two explanations is correct. Instead, if both are supported by bodies of evidence, we need to pinpoint the explanatory standards that underlie each evolutionary explanation (i.e., why researchers consider them good explanations). Doing so will allow us to understand why they are regarded as contradictory, and how we can develop strategies for integrating them within a shared theoretical framework.

Understanding explanatory power and its criteria also means becoming aware of the fact that novel explanations are not necessarily weaker than established ones. They simply have different explanatory values compared to more orthodox 'exemplars' of good explanation in evolutionary biology. This entrenchment of explanatory standards might actually be detrimental. For example, the focus on certain evolutionary causes usually entails that others are marginalized or left unexamined (see Uller et al. 2019). However, this not only holds for SET, but also for EES. The fact that some fields like systems biology and paleontology have not been sufficiently 
Table 2 Examples of standoffs between explanations of the SET and EES that address the same (or similar) evolutionary phenomena

Phenomenon addressed by EES and SET

Similar body shapes and coloration of cichlids inhabiting Lake Malawi and Lake Tanganyika

Limb lateralization preferences across vertebrate species

Flyway evolution of morphologically distinct subspecies of Red Knot Calidris canutus

Coat color evolution of Peromyscus polionotus populations that live on sand dunes or inland terrain

Evolution of heavy pigmentation of water fleas that thrive in ponds at high latitudes

Early domestication of animals

Patterns of phenotypic variation in the fossil record

Transition from single-celled to multi-cellular organisms or other transitions in biological individuality

Rapid evolution of animal traits that are sensitive to social environments

Variation in the hominin skeleton (early Homo genus)

Evolution of human language

Evolution of several feats of human cognition, e.g., cultural learning and powers of computation

Lactase persistence in human populations

The rise of the sickle-cell allele among certain agricultural groups in West Africa

\section{References}

Laland et al. (2014) (EES);

Wray et al. (2014) (SET)

Michel et al. (2018)

Piersma (2011)

Uller and Helanterä (2019)

Uller et al. (2019)

Smith (2016)

Jackson (2020)

Uller et al. (2019)

Bailey et al. (2018)

Antón and Kuzawa (2017)

Suman (2018)

Fuentes (2018)

Scott-Phillips et al. (2014)

O'Brien and Laland (2012)

References listed as 'EES' and 'SET', respectively, provide EES- and SET-style explanations for a shared phenomenon. All other references provide reviews of both opposing explanations of a certain phenomenon

integrated into the explanatory framework of EES might be due to the fact that these fields prioritize explanatory standards different from exemplars of good explanation accepted by EES (see Huang 2012; Fábregas-Tejeda and Vergara-Silva 2018b; but see Jackson 2020).

As well as showing how certain explanations rank according to particular explanatory values, our analysis suggests a way forward for theoretical integration in evolutionary biology. If two explanations achieve different explanatory standards (such as low or high precision, idealization, sensitivity, or proportionality), it will be harder to integrate them, due to the epistemological and methodological choices that lie behind these values. One illustration of this comes from the criteria that guide model organism choice. SET often favors organisms that uphold its explanatory interests in mechanistic genetic detail. However, many of these model organisms exhibit little precision in the descriptions of their developmental and organismal levels of organization. They were originally chosen due to their invariant body plans, low levels of developmental plasticity, and ease of genetic manipulation (e.g., C. elegans). Since EES defenders increasingly adopt new non-model organisms, such as Ontophagus beetles, that fit their explanatory interests in various developmental and niche 
construction processes better (see Minelli and Baedke 2014), these choices make it harder to align their data and methodologies with those of SET.

Along with general differences between explanatory values in SET and EES, there may be more fine-grained distinctions between SET and specific research areas within the EES. For instance, explanations of niche construction often focus on organismal (especially behavioral) levels. Doing so idealizes the complexity of other levels of organization, which commonly leads to less precise but highly stable explanations. One example is the explanation of how cattle-farming and milk consumption fed into the spread of lactase persistence (O'Brien and Laland 2012). Since at least some of the explanatory standards of niche construction theory (such as low precision and low sensitivity) seem to partially overlap with those of some SET-derived explanations, it would be worth investigating whether EES and SET could be integrated via common ground in niche construction theory. This similarity of standards could also be a reason for why there are a number of claims that niche construction is theoretically orthodox and could easily be integrated into SET (e.g., Wray et al. 2014; Svensson 2018).

In addition, our framework can also be applied for internal comparisons within EES (or even SET). Take, for example, research on developmental bias in horned dung beetles in the genus Onthophagus. These studies try to integrate explanations of (a) gene regulatory networks that pattern specific body regions, (b) plastic developmental mechanisms that coordinate environmental responses, and (c) developmental symbioses and niche construction that enable organisms to build assemblages and to modify their own selective environments (see Hu et al. 2020; Schwab et al. 2019). Such attempts at complex integration within EES might be problematic if there are different explanatory standards at work in the relevant explanations involving niche construction, inclusive inheritance, developmental bias and plasticity, as well as microbiomes. For instance, explanations of developmental bias that highlight the role of gene regulatory networks often seek highly precise explanations (Love 2018; Uller et al. 2018). In contrast, research on inclusive inheritance through microbiome transmission often favors explanations with low precision and many idealizations (see for discussion Roughgarden et al. 2018: 57).

We have presented a general contrastive framework suitable for evaluating the goodness of scientific explanations. It is able to distinguish why and when explanations of the EES are better than prevailing SET explanations. Our framework also suggests that there might be an overlooked diversity of explanatory standards within EES, and that making different areas of research coherent requires an assessment of the standards that operate within each contributing field. Insights into the interplay of these explanatory criteria might lead to the breakdown of gridlocked debates in evolutionary biology. Rather than emphasizing the accumulation of more evidence for either explanatory side, our framework encourages the detailed analysis of the epistemological and methodological accompaniments to different explanatory standards, and looks at how different virtues might be traded off against one another. The very existence of such trade-offs suggests the potential for integration of SET and EES, as different explanatory values can be emphasized for different purposes. We propose this framework as a helpful tool for evolutionary biology and similarly 
divided fields to become aware of the epistemic challenges of theoretical integration that lie ahead.

Acknowledgements We thank Tobias Uller, Daniel Brooks, Helmut Pulte, and Fátima Sofía ÁvilaCascajares, as well as the anonymous reviewers and the editor, Maureen A. O'Malley, for constructive comments on earlier versions of this paper. Moreover, we thank the session audiences at the workshop 'The Extended Evolutionary Synthesis: Philosophical and Historical Dimensions' in Bochum (Germany, 2019) and members of the Institute for the History and Philosophy of Science and Technology (IHPST), Paris, for feedback on presentations on this topic. We gratefully acknowledge financial support from the German Research Foundation (DFG; project no. BA 5808/1-1) (JB), as well as from the LabExchange program, Ruhr University Bochum, and the National Council of Science and Technology (CONACyT, Mexico) through Posgrado en Filosofía de la Ciencia, UNAM (AFT). Open Access funding provided by Projekt DEAL.

Open Access This article is licensed under a Creative Commons Attribution 4.0 International License, which permits use, sharing, adaptation, distribution and reproduction in any medium or format, as long as you give appropriate credit to the original author(s) and the source, provide a link to the Creative Commons licence, and indicate if changes were made. The images or other third party material in this article are included in the article's Creative Commons licence, unless indicated otherwise in a credit line to the material. If material is not included in the article's Creative Commons licence and your intended use is not permitted by statutory regulation or exceeds the permitted use, you will need to obtain permission directly from the copyright holder. To view a copy of this licence, visit http://creativecommons.org/ licenses/by/4.0/.

\section{References}

Antón SC, Kuzawa CW (2017) Early Homo, plasticity and the extended evolutionary synthesis. Interface Focus 7:20170004. https://doi.org/10.1098/rsfs.2017.0004

Baedke J (2017) Expanding views of evolution and causality. J Gen Philos Sci 48:591-594

Baedke J (2018) Above the gene, beyond biology: towards a philosophy of epigenetics. University of Pittsburgh Press, Pittsburgh

Baedke J (2019) O organism, where art thou? Old and new challenges for organism-centered biology. J Hist Biol 52:293-324

Bailey NW, Marie-Orleac L, Moore AJ (2018) Indirect genetic effects in behavioral ecology: does behavior play a special role in evolution? Behav Ecol 29(1):1-11

Bettinger RL (1993) Hunter-gatherers: archaeological and evolutionary theory. Springer, New York

Blanchard T (2018) Explanatory abstraction and the Goldilocks problem: interventionism gets things just right. Br J Philos Sci 1:1. https://doi.org/10.1093/bjps/axy030

Brigandt I (2010) The epistemic goal of a concept: accounting for the rationality of semantic change and variation. Synthese 177:19-40

Craig L (2010) The so-called extended synthesis and population genetics. Biol Theory 5:117-123

Crupi V, Tentori K, Gonzalez M (2007) On Bayesian measures of evidential support: theoretical and empirical issues. Philos Sci 74:229-252

Dickins TE, Rahman Q (2012) The extended evolutionary synthesis and the role of soft inheritance in evolution. Proc Biol Sci 279:2913-2921

Fábregas-Tejeda A (2019) New perspectives on theory change in evolutionary biology. J Gen Philos Sci 50(4):573-581

Fábregas-Tejeda A, Vergara-Silva F (2018a) The emerging structure of the extended evolutionary synthesis: where does Evo-Devo fit in? Theory Biosci 137(2):169-184

Fábregas-Tejeda A, Vergara-Silva F (2018b) Hierarchy theory of evolution and the extended evolutionary synthesis: some epistemic bridges, some conceptual rifts. Evol Biol 45:127-139

Franklin-Hall LR (2016) High-level explanation and the interventionist's 'variables problem'. Br J Philos Sci 67(2):553-577 
Fuentes A (2018) Evolution, animal behavior, culture, and the human mind. Am J Psychol 131(4):517-522

Futuyma DJ (2017) Evolutionary biology today and the call for an extended synthesis. Interface Focus 7(5):20160145. https://doi.org/10.1098/rsfs.2016.0145

Gremillion KJ, Barton L, Piperno DR (2014) Particularism and the retreat from theory in the archaeology of agricultural origins. Proc Natl Acad Sci 111(17):6171-6177

Griffiths P, Stotz K (2013) Genetics and philosophy: an introduction. Cambridge University Press, Cambridge

Guerra-García A, Piñero D (2017) Current approaches and methods in plant domestication studies. Bot Sci 95:345-362

Haig D (2011) Lamarck ascending! Philos Theor Biol 3:e204. https://doi.org/10.3998/ptb.69590 04.0003 .004

Hawkes K, O’Connell JF (1992) On optimal foraging models and subsistence transitions. Curr Anthropol 33:63-65

Hu Y, Linz DM, Parker ES, Schwab DB, Casasa S, Macagno ALM, Moczek AP (2020) Developmental bias in horned dung beetles and its contributions to innovation, adaptation, and resilience. Evol Dev 22(1-2):165-180

Huang S (2012) The molecular and mathematical basis of Waddington's epigenetic landscape: a framework for post-Darwinian biology? BioEssays 34(2):149-157

Jablonka E (2017) The evolutionary implications of epigenetic inheritance. Interface Focus 7:20160135. https://doi.org/10.1098/rsfs.2016.0135

Jablonka E, Lamb MJ (2014) Evolution in four dimensions, Revised edn. MIT Press, Cambridge

Jablonka E, Raz G (2009) Transgenerational epigenetic inheritance: prevalence, mechanisms, and implications for the study of heredity. Q Rev Biol 84:131-176

Jackson ISC (2020) Developmental bias in the fossil record. Evol Dev 22(1-2):88-102

Kovaka K (2019) Underdetermination and evidence in the developmental plasticity debate. Br J Philos Sci 70(1):127-152

Laland KN, Odling-Smee J, Feldman MW (2005) On the breadth and significance of niche construction: a reply to Griffiths, Okasha and Sterelny. Biol Philos 20(1):37-55

Laland KN, Sterelny K, Odling-Smee J et al (2011) Cause and effect in biology revisited: is Mayr's proximate-ultimate dichotomy still useful? Science 334:1512-1516

Laland KN, Odling-Smee J, Hoppitt W, Uller T (2013) More on how and why: a response to commentaries. Biol Philos 28(5):793-810

Laland K, Uller T, Feldman MW et al (2014) Does evolutionary theory need a rethink? Nature 514:161-164

Laland K, Uller T, Feldman MW et al (2015) The extended evolutionary synthesis: its structure, assumptions and predictions. Proc R Soc Lond B Biol Sci 282:20151019. https://doi.org/10.1098/ rspb.2015.1019

Laland K, Matthews B, Feldman MW (2016) An introduction to niche construction theory. Evol Ecol 30:191-202

Lewens T (2019) The extended evolutionary synthesis: what is the debate about, and what might success for the extenders look like? Biol J Linn Soc 127(4):707-721

Lins Neto EM, Albuquerque U (2018) Theories of niche construction and optimal foraging: weaknesses and virtues in understanding the early stages of domestication. Ethnobiol Conserv 7:2238-4782

Love AC (2010) Rethinking the structure of evolutionary theory for an extended synthesis. In: Pigliucci M, Müller GB (eds) Evolution: the extended synthesis. MIT Press, Boston, pp 403-441

Love AC (2018) Developmental Mechanisms. In: Glennan S, Illari P (eds) The Routledge handbook of the philosophy of mechanisms and mechanical philosophy. Routledge, New York, pp 332-347

Mayr E (1961) Cause and effect in biology. Science 134(3489):1501-1506

Mesoudi A, Blanchet S, Charmantier A et al (2013) Is non-genetic inheritance just a proximate mechanism? A corroboration of the extended evolutionary synthesis. Biol Theory 7:189-195

Meyer RS, Purugganan MD (2013) Evolution of crop species: genetics of domestication and diversification. Nat Rev Genet 14(12):840-852

Meyer RS, DuVal AE, Jensen HR (2012) Patterns and processes in crop domestication: an historical review and quantitative analysis of 203 global food crops. New Phytol 196(1):29-48

Michel GF, Babik I, Nelson EL et al (2018) Evolution and development of handedness: an evo-devo approach. Prog Brain Res 238:347-374 
Minelli A, Baedke J (2014) Model organisms in evo-devo: promises and pitfalls of the comparative approach. Hist Philos Life Sci 36(1):42-59

Moczek AP (2019) An evolutionary biology for the twenty-first century. In: Fusco G (ed) Perspectives on evolutionary and developmental biology. Padova University Press, Padova, pp 23-27

Mohlenhoff KA, Coltrain JB, Codding BF (2015) Optimal foraging theory and niche-construction theory do not stand in opposition. Proc Natl Acad Sci 112(24):E3093. https://doi.org/10.1073/pnas.15076 37112

Mueller NG, Fritz GJ, Patton P et al (2017) Growing the lost crops of eastern North America's original agricultural system. Nat Plants 3(7):17092. https://doi.org/10.1038/nplants.2017.92

Müller GB (2007) Six memos for evo-devo. In: Laubichler MD, Maienschein J (eds) From embryology to evo-devo. MIT Press, Cambridge, pp 499-524

Müller GB (2017) Why an extended evolutionary synthesis is necessary. Interface Focus 7(5):20170015. https://doi.org/10.1098/rsfs.2017.0015

Müller GB, Pigliucci M (2010) Extended synthesis: theory expansion or alternative? Biol Theory 5(3):275-276

Nathan MJ (2012) The varieties of molecular explanation. Philos Sci 79:233-254

Neander K (1995) Pruning the tree of life. Br J Philos Sci 46:59-80

Nettle D, Gibson MA, Lawson DW, Sear R (2013) Human behavioral ecology: current research and future prospects. Behav Ecol 24(5):1031-1040

Noble D (2011) Neo-Darwinism, the modern synthesis and selfish genes: are they of use in physiology? J Physiol 589:1007-1015

O’Brien MJ, Laland KN (2012) Genes, culture, and agriculture. Curr Anthropol 53(4):434-470

Odling-Smee FJ, Laland KN, Feldman M (2003) Niche construction. Princeton University Press, Princeton

Oyama S, Griffiths PE, Gray RD (eds) (2001) Cycles of contingency. MIT Press, Cambridge

Pennisi E (2008) Modernizing the modern synthesis. Science 321:196-197

Peterson T, Müller GB (2016) Phenotypic novelty in EvoDevo: the distinction between continuous and discontinuous variation and its importance in evolutionary theory. Evol Biol 43:314-333

Piersma T (2011) Flyway evolution is too fast to be explained by the modern synthesis: proposals for an 'extended' evolutionary research agenda. J Ornithol 152(1):151-159

Pigliucci M (2007) Do we need an extended evolutionary synthesis? Evolution 61:2743-2749

Pigliucci M (2008) The proper role of population genetics in modern evolutionary theory. Biol Theory 3(4):316-324

Pigliucci M, Müller GB (eds) (2010a) Evolution: the extended synthesis. MIT Press, Boston

Pigliucci M, Müller GB (2010b) Elements of an extended evolutionary synthesis. In: Pigliucci M, Müller GB (eds) Evolution: the extended synthesis. MIT Press, Boston, pp 3-17

Piperno DR (2017) Assessing elements of an extended evolutionary synthesis for plant domestication and agricultural origin research. Proc Natl Acad Sci 114:6429-6437

Piperno DR et al (2015) Teosinte before domestication: experimental study of growth and phenotypic variability in late pleistocene and early holocene environments. Quat Int 363:65-77

Potochnik A (2017) Idealization and the aims of science. University of Chicago Press, Chicago

Raerinne J, Baedke J (2015) Exclusions, explanations, and exceptions: on the causal and lawlike status of the competitive exclusion principle. Philos Theor Biol 7:e602. https://doi.org/10.3998/ptb.69590 04.0007 .002

Roughgarden J, Gilbert SF, Rosenberg E et al (2018) Holobionts as units of selection and a model of their population dynamics and evolution. Biol Theory 13(1):44-65

Schaffer J (2005) Contrastive causation. Philos Rev 114:327-358

Schlichting CD, Wund MA (2014) Phenotypic plasticity and epigenetic marking: an assessment of evidence for genetic accommodation. Evolution 68:656-672

Schwab DB, Casasa S, Moczek AP (2019) On thereciprocally causal and constructive nature of developmental plasticity and robustness. Front Genet 9:735. https://doi.org/10.3389/fgene.2018.00735

Scott-Phillips TC, Laland KN, Shuker DM et al (2014) The niche construction perspective: a critical appraisal. Evolution 68(5):1231-1243

Smith BD (2015) A comparison of niche construction theory and diet breadth models as explanatory frameworks for the initial domestication of plants and animals. J Archaeol Res 23(3):215-262

Smith BD (2016) Neo-Darwinism, niche construction theory, and the initial domestication of plants and animals. Evol Ecol 30(2):307-324

Sober E (1984) The nature of selection. University of Chicago Press, Chicago 
Sober E (1988) Apportioning causal responsibility. J Philos 85(6):303-318

Sterelny K (2011) From hominins to humans: how sapiens became behaviourally modern. Philos Trans R Soc B 366:809-822

Sultan SE (2017) Developmental plasticity: re-conceiving the genotype. Interface Focus 7:20170009. https://doi.org/10.1098/rsfs.2017.0009

Suman F (2018) Integrative and separationist perspectives: understanding the causal role of cultural transmission in human language evolution. Biol Theory 13(4):246-260

Svensson EI (2018) On reciprocal causation in the evolutionary process. Evol Biol 45(1):1-14

Tanghe KB, De Tiège A, Pauwels L, Blancke S, Braeckman J (2018) What's wrong with the modern evolutionary synthesis? A critical reply to Welch. Biol Philos 33:23

Uller T, Helanterä H (2019) Niche construction and conceptual change in evolutionary biology. Br J Philos Sci 70(2):351-375

Uller T, Laland K (eds) (2019) Evolutionary causation: biological and philosophical reflections. MIT Press, Cambridge

Uller T, Moczek AP, Watson RA et al (2018) Developmental bias and evolution: a regulatory network perspective. Genetics 209:949-966

Uller T, Feiner N, Radersma R et al (2019) Developmental plasticity and evolutionary explanations. Evol Dev. https://doi.org/10.1111/ede.12314

Weitzel EM, Codding BF (2016) Population growth as a driver of initial domestication in Eastern North America. R Soc Open Sci 3(8):160319. https://doi.org/10.1098/rsos.160319

Welch J (2017) What's wrong with evolutionary biology? Biol Philos 32:263-279

Woodward J (2003) Making things happen. Oxford University Press, Oxford

Woodward J (2010) Causation in biology: stability, specificity, and the choice of levels of explanation. Biol Philos 25:287-318

Woodward J (2018) Explanatory autonomy: the role of proportionality, stability, and conditional irrelevance. Synthese. https://doi.org/10.1007/s11229-018-01998-6

Wray GA, Hoekstra HE, Futuyma DJ et al (2014) Does evolutionary theory need a rethink? Nature 514:161-164

Yablo S (1992) Mental causation. Philos Rev 101:245-280

Ylikoski P, Kuorikoski J (2010) Dissecting explanatory power. Philos Stud 148(2):201-219

Zeder MA (2015) Core questions in domestication research. Proc Natl Acad Sci 112(11):3191-3198

Zeder MA (2017) Domestication as a model system for the extended evolutionary synthesis. Interface Focus 7(5):20160133. https://doi.org/10.1098/rsfs.2016.0133

Zeder MA (2018) Why evolutionary biology needs anthropology: evaluating core assumptions of the extended evolutionary synthesis. Evol Anthropol 27:267-284

Publisher's Note Springer Nature remains neutral with regard to jurisdictional claims in published maps and institutional affiliations. 